\title{
Model for simulation of photo-induced charges inside the hybrid LC cells
}

\author{
P. Moszczyński, A. Walczak, P. Marciniak \\ Faculty of Cybernetics, Military University of Technology, Kaliski Str.2, 00-908 Warsaw, Poland.
}

Received February 14, 2013; accepted March 28, 2013; published March 31, 2013

\begin{abstract}
A mathematical model is presented for a quantitative description of the liquid crystal (LC) layer deformation inside an asymmetric hybrid liquid crystalline cell. Asymmetry means that one aligning layer in the cell is composed as a photoconductive polymer PVK:TNF while the second is covered with a common aligning material (polyimide). The LC waveguide arises along the laser beam path propagated in plane of the LC layer (refraction of light is not presented). In the presented model we proved that an LC layer deformation is caused by the synergy of electric fields: driving field, space charge field, and fields of the surface charge cumulated on facing borders PVK:TNF/ITO and PVK:TNF/LC.
\end{abstract}

When exposed to light of appropriate wavelength, the command surface in a hybrid cell induces a reversible change of orientation in the LC layer situated next to the command surface (see Fig.1). Such a change of the LC layer orientation can create an optical waveguide along the way of an external beam propagated along the layer $[1,2]$. In this letter we present how to calculate an additional control field that is derived from a photoinduced space-charge inside the photoconductive command surfaces.

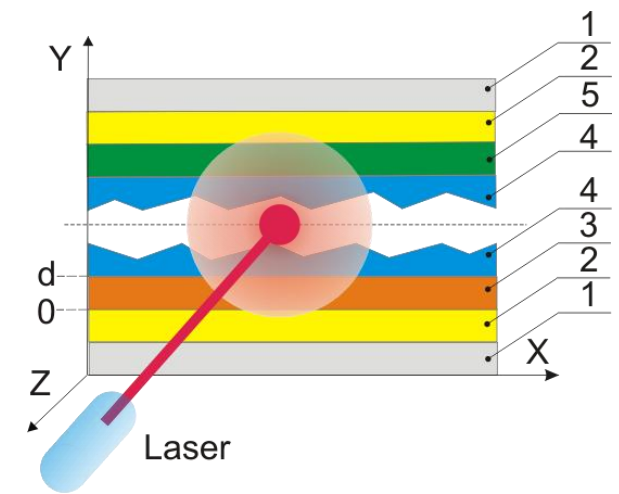

Fig.1. LC cell configuration: 1 - glass, 2- ITO layer, 3- photoconductive polymer layer, 4- LC layer, 5 - polyimide layer. The parameter $\mathrm{d}$ is a thickness of the PVK:TNF layer.

The profile of voltage applied to the polymer layer is obtained as a result of the integrate electric field inside this layer. This electric field is a sum of the external field and photo induced charges field and the charge from the layer borders.

The generation and transport of the carriers model inside a photoconductive polymer are described by a simplified Kukhtarev [3, 4] and Schildkraut model [5, 6]. Our model extends descriptions of the space-charge field formed inside photoconductive materials.

It is well-known that due to the irregular intensity of light inside a photoconductive polymer layer induces a spacecharge effect. We propose a two-dimensional model of charge movement and we use an electrostatic method of mirror images to calculate results. We assume exponential field decay from the surface charge inside the ITO layer. The formulae of a math problem are presented below.

$$
\begin{gathered}
\frac{\partial p}{\partial t}=\varphi I(x)\left(N_{A}-N_{A}^{-}\right)-\mu_{p} p N_{A}^{-}-r\left(T_{H}-T_{H}^{+}\right)+ \\
+\gamma p T_{H}^{+}+\operatorname{div}(\vec{\jmath}) \\
\frac{\partial N_{A}^{-}}{\partial t}=\varphi I(x)\left(N_{A}-N_{A}^{-}\right)-\mu_{p} p N_{A}^{-} \\
\frac{\partial T_{H}^{+}}{\partial t}=r\left(T_{H}-T_{H}^{+}\right)+\gamma p T_{H}^{+} \\
\vec{J}=\mu_{p} p\left(\vec{E}+\overrightarrow{E_{s}}\right)+\frac{k T}{e} \nabla p \\
\nabla \cdot \vec{E}=e / \varepsilon_{p}\left(p-N_{A}^{-}+T_{H}^{+}\right)
\end{gathered}
$$

For a numerical solution of (1)-(5) we assume the border conditions (6)-(9), where parameter d means the thickness of the polymer layer.

$$
\begin{gathered}
\lim _{y \rightarrow 0^{-}}\left(\begin{array}{c}
E_{x} \\
E_{y}
\end{array}\right)=\left(\begin{array}{l}
0 \\
0
\end{array}\right) \quad \lim _{y \rightarrow 0^{+}}\left(\begin{array}{c}
E_{x} \\
E_{y}
\end{array}\right)=\left(\begin{array}{c}
0 \\
\varepsilon_{p} E_{y}
\end{array}\right) \\
-\varepsilon_{p} E_{y}=4 \pi \sigma_{I T O / P} \\
\lim _{y \rightarrow d^{-}}\left(\begin{array}{c}
E_{x} \\
E_{y}
\end{array}\right)=\left(\begin{array}{c}
E_{x} \\
\varepsilon_{p} E_{y}
\end{array}\right) \lim _{y \rightarrow d^{+}}\left(\begin{array}{c}
E_{x} \\
E_{y}
\end{array}\right)=\left(\begin{array}{c}
E_{x} \\
\varepsilon_{L C} E_{y}
\end{array}\right) \\
\varepsilon_{p} E_{y}-\varepsilon_{L C} E_{y}=4 \pi \sigma_{P / L C}
\end{gathered}
$$

The transport of the charge along two perpendicular directions (X, Y see Fig.1) inside the polymer structure is taken into account. The equations from (1) to (5) describe the transport and generation of the carriers inside 
photoconductive media. The first three illustrate the principle of charge conservation and how the concentration of hole $\mathrm{p}$, ionized acceptor impurity $N_{A}^{-}$and charged trap $T_{H}^{+}$varies in time. We use trap levels from the Schildkraut theory to the followed calculations. We apply new constants: densities of trap $T_{H}$, trapping and detrapping rates (constants: $r$ and $\gamma$ in (3)). The current density of holes $\mathbf{j}$ is determined from (4), which includes the part of drift in the field and the part of carriers diffusion. Symbols k, e and T are the Boltzmann constant, electron charge and temperature, respectively. Equation (5) is a Gauss equation.

We assumed that only holes mobility $\mu_{p}$ is different than zero. The concentration change resulting from the diffusion and drift of charges is described as a divergence part in the first equation. The first two parts of equation (1-2) describe photogeneration where $\varphi$ is the photogeneration efficiency.

To construct the boundary conditions for 2-dimensional Gauss equation (5) we use the method of mirror images. The density of the charge $\sigma_{\text {ITO/P }}$ from the ITO/polymer boundary was calculated by assuming it is a metal /dielectric boundary. The density of the charge $\sigma_{P / L C}$ from a photoconductive polymer/LC boundary, however, was obtained from the model of a dielectric/dielectric boundary. Constants: $\varepsilon_{P}$ and $\varepsilon_{L C}$ are the permittivity of the photoconductive polymer and effective permittivity liquid crystalline, respectively (Fig.2).

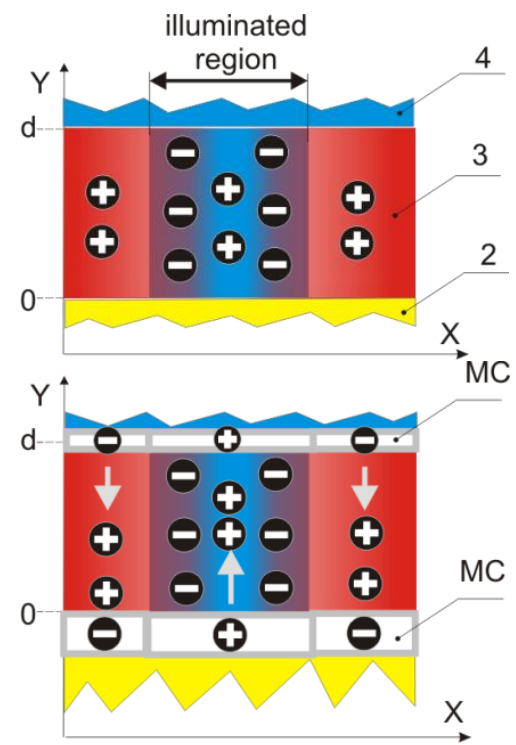

a) Generation and diffusion of holes inside the polymer layers as a result of illumination. Signs (+) and (-) illustrate concentrations of the charges inside the structure.

b) Value of charges on the borders of the polymer layer is different. This asymmetry causes migration of charges inside the polymer.

Fig.2. Idea of the mirror images method for the polymer layer (3) and neighbouring layers (LC and ITO).

The space charge density on the border between ITOpolymer and polymer-LC layers will be calculated using (10) and (11) formulas adequately.

$$
\begin{aligned}
& \sigma_{\text {IтO /P }}(x) \\
& =\int_{\alpha=-\infty}^{\infty} \int_{y=0}^{d}-\frac{Q(x, y)}{\varepsilon_{p}\left((\alpha-x)^{2}+y^{2}\right)^{\frac{3}{2}}} d y d \alpha \\
& \ldots+\int_{\alpha=-\infty}^{\infty}-\frac{\sigma_{P / L C}(\alpha)}{2 \pi \varepsilon_{p}} \frac{y}{\left((\alpha-x)^{2}+y^{2}\right)^{\frac{3}{2}}} d \alpha \\
& \begin{array}{c}
\sigma_{P / L C}(x)=\int_{\begin{array}{c}
\alpha=-\infty \\
d=0 \\
d-y
\end{array}}^{\infty}-\frac{\varepsilon_{L C}-\varepsilon_{p}}{\varepsilon_{p}\left(\varepsilon_{L C}+\varepsilon_{p}\right)} \frac{Q(x, y)}{2 \pi} \times \\
\ldots \times \frac{\left((\alpha-x)^{2}+(d-y)^{2}\right)^{\frac{3}{2}}}{((y) d \alpha+\cdots}
\end{array} \\
& +\int_{\alpha=-\infty}^{\infty}-\frac{\varepsilon_{L C}-\varepsilon_{p}}{\varepsilon_{p}\left(\varepsilon_{L C}+\varepsilon_{p}\right)} \frac{\sigma_{I T O / P}(\alpha)}{2 \pi \varepsilon_{p}} \frac{d}{\left((\alpha-x)^{2}+d^{2}\right)^{\frac{3}{2}}} d \alpha \\
& Q(x, y)=\frac{e\left(p(x, y)-N_{A}^{-}(x, y)+T_{H}^{+}(x, y)\right)}{2 \pi \varepsilon_{p}}
\end{aligned}
$$

Therefore to calculate the control field of deformation of the liquid crystal, we can now use the formula below.

$$
U_{L C}(x)=\int_{0}^{d} E_{y}(x, y) d y
$$

The reorientation effect of the molecules of $\mathrm{LC}$ is a consequence of the existing space-charge inside the polymer layer. This charge is separated as a result of interaction between charges on the boundaries: polymer/LC and polymer/ITO. Holes are transported to the LC layer and impurities are accumulated near the ITO boundary. Voltage arising in the polymer will be applied to the LC and polyimide. An ITO medium is a n-type semiconductor with a very high value of the work function and a very high concentration of electrons. At the surfaces of this material a positive charge with a high value arises and prevents electrons movement. This charge on the surface is the source of electric field $E_{S}$ taken into account in (4).

In our simulation, we assume that $E_{S}$ exponentially decreases with a distance to the surface. The source of $E$ field (4) are the space-charges and boundary charges.

The PVK:TNF mixture inside a hybrid cell with a molar concentration 1:0.2 is a p-type photoconductive semiconductor [7]. Illuminated PVK:TNF is a source of mobile holes and static acceptor impurities. Induced holes diffuse into non-illuminated regions (Fig.2a.). Therefore the illuminated area is negatively charged and the tiled area-positively. 
The jump of an electrostatic induction vector from the polymer/ITO boundary and is much higher than the one from the polymer/LC. So mirror charges appear inside the polymer layer. This produces a drift of the charge compensated by diffusion. The asymmetric distribution of the charge on the boundary causes holes shift in the polymer (Fig.2b.). On the boundary of the illuminated area, a positive charge is induced. The holes relocate to the LC boundary. Inside the dark area on the boundaries negative charges are induced and relocate to the ITO boundary.

Numerical results are obtained with a finite-difference method (FDM) and are shown in Figs. 3-4. First, we assume the Gaussian profile of illumination function (Fig. 3).
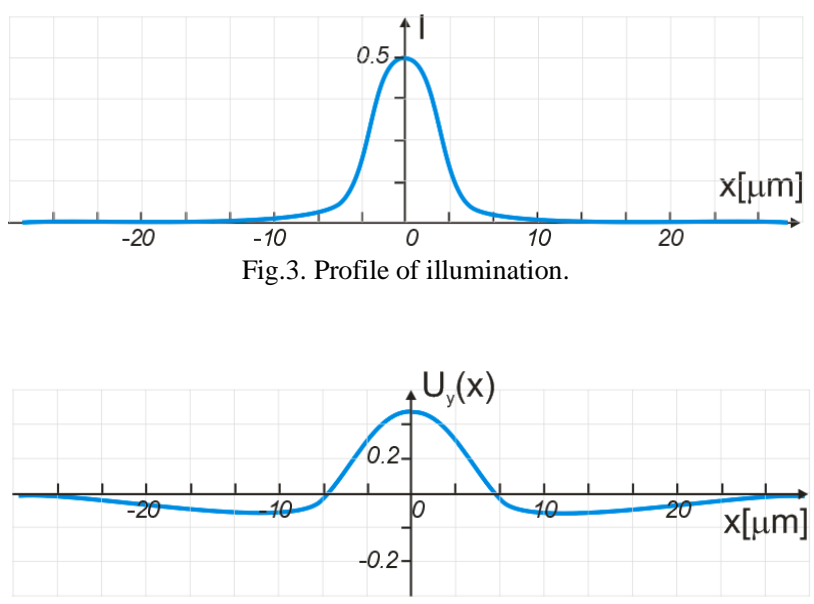

Fig.4. Result of the numerical calculation of $U_{y}$.

The results of the simulation prove that the generation of a space charge inside the illuminated layer decreases the voltage inside the photoconductive polymer layer. Due to the fact that the voltage between ITO electrodes must be the same as inside the cell, the compensating voltage will be accumulated into the LC layer.

The presented model gives possible simulation processes on the border of a liquid crystal and a photo-generated semiconductor medium. It is a full mathematical model of the effects produced on the border between a nematic liquid crystal and a photo induced semiconductor.

The proposed model allows one to create an effective simulation of complicated phenomena, and allows explaining the differences between the observed results for various LC substances. A detailed model of a space charge field generated inside the PVK:TNF layer has been shown. We also proved, that the surface charge is an important component of the model because the PVK:TNF layer is of very low thickness. As far as authors know this is the first complete and effective simulation in a relatively long history of hybrid LC cells exploration. It turned out that boundary phenomena have an important meaning in the presented model

\section{References}

[1] A. Walczak, P. Moszczyński, E. Nowinowski-Kruszelnicki, Mol. Cryst. Liq. Cryst. 559(1), 186 (2012).

[2] A. Walczak, E. Nowinowski-Kruszelnicki, Opt. Eng. 47(3), 035402 (2008).

[3] N. Kukhtarev, V. Markov, S. Odulov, M. Soskin, V. Vinetskii, Ferroelect. 22, 949 (1979).

[4] N. Kukhtarev, T. Kukhtareva, Proc. IEEE, 87(11), 1857 (1999).

[5] J.S. Schildkraut, Y. Cui, J. Appl. Phys. 72 (11), 5055 (1992).

[6] J.S. Schildkraut, A.V. Buettner, J. Appl. Phys. 72 (5), 1888 (1992).

[7] W.D. Gill, J. Appl. Phys. 43(12), 5033 (1972). 